\title{
XLVIII. Note on the action of coupled circuits and mechanical analogies
}

\section{H.C. Plummer}

To cite this article: H.C. Plummer (1917) XLVIII. Note on the action of coupled circuits and mechanical analogies, Philosophical Magazine Series 6, 34:204, 510-517, DOI: $10.1080 / 14786441708565208$

To link to this article: http://dx.doi.org/10.1080/14786441708565208

曲 Published online: 08 Apr 2009.

Submit your article to this journal $₫$

Џ Article views: 2

Q View related articles $₫$ 
XLVIII. Note on the Action of Coupled Circuits and Mechanical Analogies. By H. C. Plummer* .

1. THE paper by Prof. Barton and Miss Browning (p. 246) suggests a doubt whether a simple electrical problem really is made easier for the average student by a complicated mechanical analogy. It seems as if the avowed purpose is to remedy the difficulty of one problem not only by setting another but by requiring the relation between the two to be understood. Yet surely the real difficulty begins, and almost ends, in the electrical problem, in establishing the fundamental equations. The mechanical analogy does not assist in this but only in the subsequent interpretation.

Curiously enough, the authors do not seem to have presented their analogies in the clearest light. Thus, for example, their equations (27) and (28) may be re-written in the form

$$
\begin{aligned}
& (\mathrm{P}+\mathrm{Q}+\beta \mathrm{P}) \frac{d^{2} y}{d t^{2}}+(\mathrm{P}+\mathrm{Q}) g l^{-1} y=-\beta \mathrm{Q} \frac{d^{2} z}{d t^{2}}, \\
& (\mathrm{P}+\mathrm{Q}+\beta \mathrm{Q}) \frac{d^{2} y}{d t^{2}}+(\mathrm{P}+\mathrm{Q}) g l^{-1} z=-\beta \mathrm{P} \frac{d^{2} y}{d t^{2}} .
\end{aligned}
$$

The analogy is now exact, and requires

$$
\begin{aligned}
\mathrm{LR} & =\frac{l}{g} \cdot \frac{\mathrm{P}+\mathrm{Q}+\beta \mathrm{P}}{\mathrm{P}+\mathrm{Q}}, \quad \mathrm{NS}=\frac{l}{g} \cdot \frac{\mathrm{P}+\mathrm{Q}+\beta \mathrm{Q}}{\mathrm{P}+\mathrm{Q}}, \\
-\mathrm{MR} & =\frac{l}{g} \cdot \frac{\beta \mathrm{Q}}{\mathrm{P}+\mathrm{Q}}, \quad-\mathrm{MS}=\frac{l}{g} \cdot \frac{\beta \mathrm{P}}{\mathrm{P}+\mathrm{Q}},
\end{aligned}
$$

which give for the coupling

$$
\gamma^{2}=\frac{\beta^{2} \mathrm{PQ}}{(\mathrm{P}+\mathrm{Q}+\beta \mathrm{P})(\mathrm{P}+\mathrm{Q}+\beta \mathrm{Q})},
$$

more distinctly than the authors seem to have shown. At the same time $L R-N S=M(R-S)$, a limitation of the conditions apparently overlooked. The second analogy does not suffer from the same defect, but in general terms is even more complicated. The coupling may be made positive by reversing the sign of $y$ or $z$.

* Communicated by the Author. 
2. The equations of the problem may be written

$$
\begin{aligned}
& \operatorname{LR} \frac{d^{2} x}{d t^{2}}-\operatorname{MR} \frac{d^{2} y}{d t^{2}}=-x, \\
& \operatorname{MS} \frac{d^{2} x}{d t^{2}}-\mathrm{NS} \frac{d^{2} y}{d t^{2}}=y .
\end{aligned}
$$

Even from the point of view of the student, the method of normal coordinates seems the simplest in this case. For all values of $\lambda$,

Let

$$
(\mathrm{LR}+\lambda . \mathrm{MS}) \frac{d^{2} x}{d t^{2}}-(\mathrm{MR}+\lambda . \mathrm{NS}) \frac{d^{2} y}{d t^{2}}=-x+\lambda y .
$$

$$
\mathrm{MR}+\lambda . \mathrm{NS}=\lambda(\mathrm{LR}+\lambda . \mathrm{MS})
$$

and then

$$
(\mathrm{LR}+\lambda . \mathrm{MS}) \frac{d^{2}}{d t^{2}}(x-\lambda y)+(x-\lambda y)=0,
$$

so that the solution is

But the equation for $\lambda$,

$$
x-\lambda y=k \cos \left(\frac{t}{p}+\epsilon\right), \quad p^{2}=\mathrm{LR}+\lambda . \mathrm{MS} .
$$

$$
\lambda^{2} . \mathrm{MS}-\lambda(\mathrm{NS}-\mathrm{LR})-\mathrm{MR}=0 . .
$$

is a quadratic with two roots $\lambda_{1}, \lambda_{2}$. The complete solution is therefore given by

$$
\begin{array}{rlrl}
x-\lambda_{1} y & =k_{1} \cos \left(\frac{t}{p_{1}}+\epsilon_{1}\right), & x-\lambda_{2} y & =k_{2} \cos \left(\frac{t}{p_{2}}+\epsilon_{2}\right), \\
p_{1}{ }^{2} & =\mathrm{LR}+\lambda_{1} . \mathrm{MS}, \quad p_{2}{ }^{2}=\mathrm{LR}+\lambda_{2} . \mathrm{MS},
\end{array}
$$

where $2 \pi p_{1}, 2 \pi p_{2}$ are the periods. Let $2 \pi \mathrm{P}_{1}, 2 \pi \mathrm{P}_{2}$ be the natural (uncoupled) periods, so that $\mathrm{P}_{1}{ }^{2}=\mathrm{L} R, \mathrm{P}_{2}{ }^{2}=\mathrm{NS}$. Then

$$
\begin{aligned}
p_{1}{ }^{2}+{p_{2}}^{2} & =2 \mathrm{LR}+\left(\lambda_{1}+\lambda_{2}\right) \mathrm{MS}=\mathrm{LR}+\mathrm{NS}, \\
p_{1}{ }^{2} p_{2}{ }^{2} & =\mathrm{LR}\left\{\mathrm{LR}+\left(\lambda_{1}+\lambda_{2}\right) \mathrm{MS}\right\}+\lambda_{1} \lambda_{2} \cdot \mathrm{M}^{2} \mathrm{~S}^{2} \\
& =\mathrm{LR} . \mathrm{NS}-\mathrm{M}^{2} \mathrm{RS},
\end{aligned}
$$

or, the coupling being given by $\mathrm{M}^{2}=\gamma^{2} . \mathrm{LN}$,

$$
p_{1}^{2}+p_{2}^{2}=\mathrm{P}_{1}^{2}+\mathrm{P}_{2}^{2}, \quad p_{1}^{2} p_{2}^{2}=\mathrm{P}_{1}^{2} \mathrm{P}_{2}^{2}\left(1-\gamma^{2}\right) . \quad \text {. }
$$

The method seems so obvious that it is probably well known even in elementary teaching. In a case like this it can be understood when the simple general theory of linear equations with oonstant coefficients is not familiar. 
3. If initially the currents are zero, $\epsilon_{1}=\varepsilon_{2}=0$; if at the same time the charges are $x=a, y=0,(2)$ give immediately

$$
x-\lambda_{1} y=\alpha \cos p_{1}^{-1} t, x-\lambda_{2} y=a \cos p_{2}^{-1} t \text {. }
$$

The equations (3) hardly exhibit the variations of $p_{1}, p_{2}$ with $\gamma$ very clearly. The quadratic in $p^{2}$,

$$
p^{4}-\left(\mathrm{P}_{1}{ }^{2}+\mathrm{P}_{2}{ }^{2}\right) p^{2}+\mathrm{P}_{1}{ }^{2} \mathrm{P}_{2}{ }^{2}\left(1-\gamma^{2}\right)=0,
$$

is no better in this respect, and its solution conveys the relations even more obscurely. There seems, therefore, to be some excuse for a graphical representation.

Fig. 1.

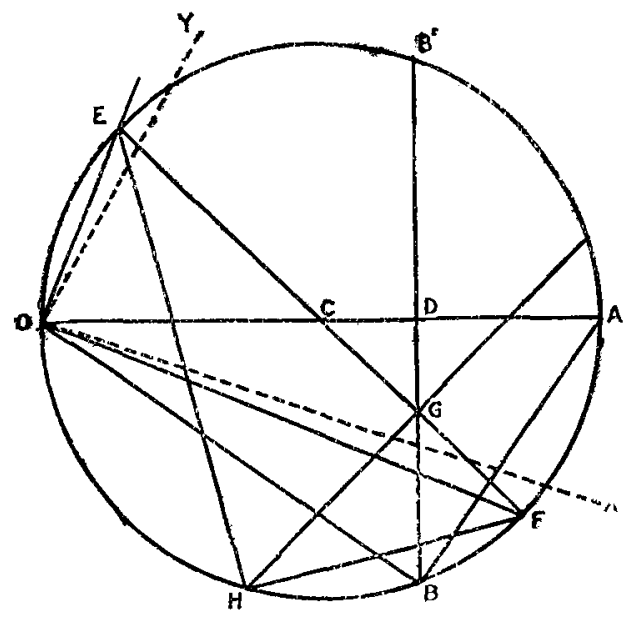

On OA, the diameter of a circle (fig. 1), let OD represent $\mathrm{P}_{1}{ }^{2}$, and $\mathrm{DA}$ represent $\mathrm{P}_{2}{ }^{2}$. The perpendicular $\mathrm{BD}$ represents $\mathrm{P}_{1} \mathrm{P}_{2}$. Take $\mathrm{G}$ on $\mathrm{BD}$ so that $G D / B D=\gamma$. Let ECGF be the diameter through $G$. Then

$$
\begin{aligned}
\mathrm{EG}+\mathrm{GF}=\mathrm{OD}+\mathrm{DA} & =\mathrm{P}_{1}{ }^{2}+\mathrm{P}_{2}{ }^{2} ; \\
\mathrm{EG} \cdot \mathrm{GF}=\mathrm{BG} \cdot \mathrm{GB}^{\prime} & =\mathrm{P}_{1} \mathrm{P}_{2}(1-\gamma) \cdot \mathrm{P}_{1} \mathrm{P}_{2}(1+\gamma) \\
& =\mathrm{P}_{1}{ }^{2} \mathrm{P}_{2}{ }^{2}\left(1-\gamma^{2}\right) .
\end{aligned}
$$

Hence EG represents $p_{1}^{2}$ and GF represents $p_{2}{ }^{2}$. Thus as the coupling increases from $D$ to $B$, the squares of the periods $\mathrm{OD}, \mathrm{DA}$ change in a way easily apprehended until one disappears and the other is represented by the diameter of the circle. 
Or again, since $B D$ represents $P_{1} P_{2}$, and $H G$ represents $p_{1} p_{2}, \mathrm{HG}$ being perpendicular to $\mathrm{EF}$,

$$
\begin{aligned}
\mathrm{OB}^{2}=\mathrm{P}_{1}{ }^{2}\left(\mathrm{P}_{1}{ }^{2}+\mathrm{P}_{2}^{2}\right), & \mathrm{BA}^{2}=\mathrm{P}_{2}{ }^{2}\left(\mathrm{P}_{1}{ }^{2}+\mathrm{P}_{2}{ }^{2}\right), \\
\mathrm{EH}^{2}=p_{1}{ }^{2}\left(p_{1}{ }^{2}+p_{2}{ }^{2}\right), & \mathrm{HF}^{2}=p_{2}{ }^{2}\left(p_{1}{ }^{2}+p_{2}{ }^{2}\right) .
\end{aligned}
$$

Hence on the same scale by which $\mathrm{P}_{1}, \mathrm{P}_{2}$ are indicated by $\mathrm{OB}, \mathrm{BA}, \mathrm{EH}$ represents $p_{1}$ and HE represents $p_{2}$. Thus if $\mathrm{OB}, \mathrm{BA}$ represent the natural periods, the periods under coupling are shown by $\mathrm{EH}, \mathrm{HF}$ and their changes can be traced in a much more instructive way than the equations alone, simple as they are, naturally suggest.

4. There is another elementary point. If $x, y$ are considered as coordinates the fundamental vibrations are perpendicular to the lines $x-\lambda_{1} y=0, x-\lambda_{2} y=0$, which are not at right angles. Let

so that (1) becomes

$$
m \lambda \sqrt{ } \mathrm{S}=\sqrt{ } \mathrm{R}
$$

$$
\mathrm{M} \sqrt{ }(\mathrm{RS})-m(\mathrm{NS}-\mathrm{LR})-m^{2} \mathrm{M} \sqrt{ }(\mathbf{R S})=0,
$$

$$
\frac{2 m}{1-m^{2}}=\frac{2 \gamma \sqrt{ }(\mathrm{LNRS})}{\mathrm{NS}-\mathrm{LR}}=\frac{\gamma \cdot \mathrm{P}_{1} \mathrm{P}_{2}}{\frac{1}{2}\left(\mathrm{P}_{2}^{2}-\overline{\mathrm{P}}_{1}^{2}\right)} .
$$

In fig. 1 this fraction is represented by $-G D / C D$, and thus $m=\tan \frac{1}{2} \mathrm{GCO}=\tan \mathrm{FOC}$. The lines

$$
y=m_{1} x, \quad y=m_{2} x
$$

are therefore represented in direction by $\mathrm{OF}, \mathrm{OE}$. But the required lines are

$$
\mathrm{R}^{\frac{1}{2}} y=m_{1} \mathrm{~S}^{\frac{1}{2}} x, \quad \mathrm{R}^{\frac{1}{2}} y=m_{2} \mathrm{~S}^{\frac{1}{2}} x,
$$

and the latter are easily derived from the former when the ratio $R / S$ is given. They are represented by $O X, O Y$ in the figure. The simple oscillations are perpendicular to $O X, O Y$, and their periods are $2 \pi p_{1}, 2 \pi p_{2}$. The resultant compound oscillations are to be found from their components perpendicular to $\mathrm{DA}, \mathrm{DB}^{\prime}$.

The fact that $O X, O Y$ are not rectangular axes is an inconvenience which can be avoided by taking other variables $\xi, \eta$ such that

$$
x=\mathrm{R}^{\frac{1}{\xi}} \xi, \quad y=\mathrm{S}^{\frac{1}{2}} \eta . \quad . \quad . \quad . .
$$

As this change of variables is independent of the coupling, it simplifies the problem without involving any complication, and can therefore be introduced with advantage at the beginning. The problem is then brought to its simplest terms as the projection of two rectangular harmonic motions 
on two other rectangular axes. The fundamental periods are shown graphically in fig. 1, and the inclination of the two sets of axes, which is now simply AOF.

5. The problem may also be considered in a slightly less elementary way. The fundamental equations may be written in the form

$$
\begin{aligned}
& \left(\mathrm{LN}-\mathrm{M}^{2}\right) \mathrm{RS} \ddot{x}=-\mathrm{NS} x-\mathrm{MR} y, \\
& \left(\mathrm{LN}-\mathrm{M}^{2}\right) \mathrm{RS} \ddot{y}=-\mathrm{MS} x-\mathrm{LR} y .
\end{aligned}
$$

Now these equations will also follow in a dynamical problem defined by the kinetic energy $\mathrm{T}$ and the potential energy $\mathrm{U}$, where

$$
\begin{aligned}
2 \mathrm{~T} & =\left(\mathrm{LN}-\mathrm{M}^{2}\right)\left(\mathrm{S} \dot{x}^{2}+\mathrm{R} \dot{y}^{2}\right), \\
2 \mathrm{U} & =\frac{\mathrm{NS}}{\mathrm{R}} x^{2}+2 \mathrm{M} x y+\frac{\mathrm{I} \mathrm{R}}{\mathrm{S}} y^{2} .
\end{aligned}
$$

The common conjugate diameters of the ellipses $2 U=1$, $\mathrm{S} x^{2}+\mathrm{R} y^{2}=1$, are

or

$$
\begin{aligned}
\mathrm{MS} x^{2}-(\mathrm{NS}-\mathrm{LR}) x y-\mathrm{MR} y^{2} & =0, \\
\left(x-\lambda_{1} y\right)\left(x-\lambda_{2} y\right) & =0,
\end{aligned}
$$

by (1). Normal coordinates are obtained by taking these as axes. But it is simpler to take $\xi, \eta$ as the variables according to (4). Then

$$
\begin{aligned}
& 2 \mathrm{~T}=\left(\mathrm{LN}-\mathrm{M}^{2}\right) \mathrm{RS}\left(\dot{\xi}^{2}+\dot{\eta}^{2}\right), \\
& 2 \mathrm{U}=\mathrm{NS} \xi^{2}+2 \mathrm{MR}^{\frac{1}{2}} \mathrm{~S}^{\frac{1}{2}} \xi \eta+\mathrm{LR} \eta^{2} .
\end{aligned}
$$

Since $\mathrm{P}_{1}^{2}=\mathrm{LR}, \mathrm{P}_{2}^{2}=\mathrm{NS}, \mathrm{M}^{2}=\gamma^{2} . \mathrm{LN}$, these : may be written

$$
\left.\begin{array}{rl}
2 \mathrm{~T} & =\left(1-\gamma^{2}\right) \mathrm{P}_{1}^{2} \mathrm{P}_{2}^{2}\left(\dot{\xi}^{2}+\dot{\eta}^{2}\right) \\
2 \mathrm{U} & =\mathrm{P}_{2}^{2} \xi^{2}+2 \gamma \cdot \mathrm{P}_{1} \mathrm{P}_{2} \xi \eta+\mathrm{P}_{1}^{2} \eta^{2}
\end{array}\right\} . . .
$$

The axes of $U$ are

$$
\gamma \cdot \mathrm{P}_{1} \mathrm{P}_{2}\left(\xi^{2}-\eta^{2}\right)=\left(\mathrm{P}_{2}^{2}-\mathrm{P}_{1}^{2}\right) \xi \eta
$$

and if these are taken as the axes of $\mathrm{X}, \mathrm{Y}$,

$$
\begin{aligned}
& 2 \mathrm{~T}=p_{1}{ }^{2} p_{2}{ }^{2}\left(\dot{\mathrm{X}}^{2}+\dot{\mathrm{Y}}^{2}\right), \\
& 2 \mathrm{U}=p_{2}{ }^{2} \mathrm{X}^{2}+p_{1}{ }^{2} \mathrm{Y}^{2},
\end{aligned}
$$

where $p_{2}^{2}+p_{1}^{2}=\mathrm{P}_{2}^{2}+\mathrm{P}_{1}^{2}, p_{1}^{2} p_{2}^{2}=\mathrm{P}_{1}^{2} \mathrm{P}_{2}^{2}\left(1-\gamma^{2}\right)$, as before, by simply comparing the last two forms of $U$. The rest of the solution follows immediately.

6. The obvious analogy suggested by (5) may just be worth noticing. On the surface

$$
2\left(1-\gamma^{2}\right) \mathrm{P}_{1}^{2} \mathrm{P}_{2}^{2} g \xi=\mathrm{P}_{2}^{2} \xi^{2}+2 \gamma \cdot \mathrm{P}_{1} \mathrm{P}_{2} \xi \eta+\mathrm{P}_{1}^{2} \eta^{2},
$$

the axis of $\zeta$ being vertical, a particle is oscillating under 
gravity in the immediate neighbourhood of the origin. It is evident that its kinetic and potential energies are proportional to $\mathrm{T}$ and $\mathrm{U}$ as defined by (5). Hence its motion presents an analogy to the problem considered. The surface is fully defined by the section on the definite plane $\zeta=\frac{1}{2} g^{-1}$, and this section is

$$
\begin{aligned}
\left(1-\gamma^{2}\right) \mathrm{P}_{1}{ }^{2} \mathrm{P}_{2}{ }^{2} & =\mathrm{P}_{2}{ }^{2} \xi^{2}+2 \gamma \cdot \mathrm{P}_{1} \mathrm{P}_{2} \xi \eta+\mathrm{P}_{1}{ }^{2} \eta^{2} \\
& =\left(\mathrm{P}_{2} \xi+\gamma \cdot \mathrm{P}_{1} \eta\right)^{2}+\left(1-\gamma^{2}\right) \mathrm{P}_{1}{ }^{2} \eta^{2} \\
& =\left(1-\gamma^{2}\right) \mathrm{P}_{2}{ }^{2} \xi^{2}+\left(\gamma \cdot \mathrm{P}_{2} \xi+\mathrm{P}_{1} \eta\right)^{2}
\end{aligned}
$$

or clearly an ellipse inscribed in the rectangle $\xi= \pm \mathrm{P}_{1}$, $\eta= \pm P_{2}$. The ellipse, which is also represented by

$$
\xi=\mathrm{P}_{1} \cos \theta, \quad \eta=-\mathrm{P}_{2} \sin (\theta+\alpha), \quad \sin \alpha=\gamma,
$$

indicates by its axes both the periods and the directions of the fundamental oscillations. The area of the ellipse, compared with its maximum, is $\cos \alpha$ or $\sqrt{ }\left(1-\gamma^{2}\right)$. In the limit the ellipse degenerates into one diagonal of the containing rectangle. The effect of the coupling is very clearly shown by the change in the shape and area of the ellipse, and the slewing round of the axes seems to show very well how the fundamental oscillations must enter into both systems dependently. The two degrees of freedom of the electrical system are represented by the displacements of a single material particle parallel to two fixed rectangular axes, and what may be lost in distinctness seems to be compensated in the very clear connexion between the two displacements. It is the natural type of all small oscillations with two degrees of freedom, and if it is not well adapted to detailed experiments it has the advantage of being most readily apprehended without any experiment at all.

Fig. 2.

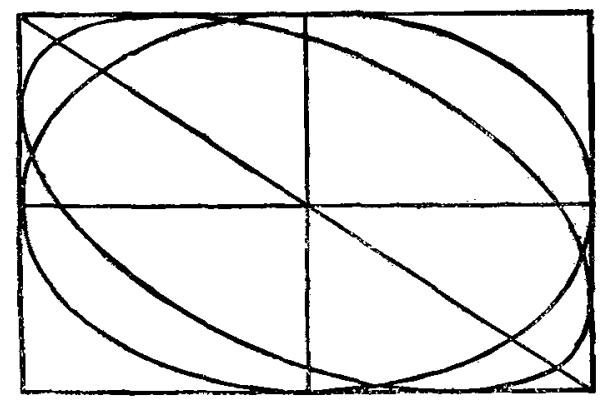

Fig. 2 shows the corresponding sections of the surfaces when $P_{1} / P_{2}=1.5$ and the coupling $\gamma$ is $0,0.5$, and 1 . 
7. The differential equations of the problem are also formally reproduced when

$$
\begin{aligned}
& 2 \mathrm{~T}=\mathrm{L} \dot{x}^{2}-2 \mathrm{M} \dot{x} \dot{y}+\mathrm{N} \dot{y}^{2}, \\
& 2 \mathrm{U}=x^{2} / \mathrm{R}+y^{2} / \mathrm{S} .
\end{aligned}
$$

These suggest the mechanism represented in fig. 3 . Here a

Fig. 3.

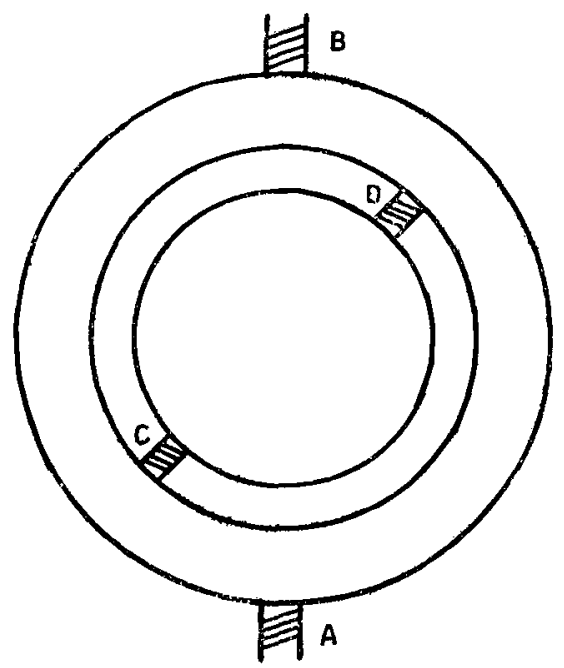

heavy ring is pivoted on an axis $A B$, about which it can turn with a spring connexion. It carries the axis $C D$ on which is pivoted a heavy concentric sphere, also with a spring attachment. If the axes $\mathrm{CD}, \mathrm{AB}$ are inclined at an angle $\theta$, while the ring rotates about $\mathrm{AB}$ through an angle $\psi$ and the sphere rotates about $C D$ through an angle $\phi$ relative to the ring, the mechanism presents a framework of Eulerian angles. The rotation of the sphere about $\mathrm{A} B$ can be resolved into components $\dot{\psi} \cos \theta$ about $\mathrm{CD}$ and $\dot{\psi} \sin \theta$ about the perpendicular axis. Hence if $\mathrm{C}$ is the moment of inertia of the sphere about any axis and $B$ is the moment of inertia of the ring about $A B$, the kinetic energy of the system is given by

$$
\begin{aligned}
2 \mathrm{~T} & =\mathrm{C}(\dot{\phi}+\dot{\psi} \cos \theta)^{2}+\mathrm{C} \dot{\psi}^{2} \sin ^{2} \theta+\mathrm{B} \dot{\psi}^{2} \\
& =\mathrm{C} \dot{\phi}^{2}+2\left(\dot{\phi} \dot{\psi} \cos \theta+(\mathrm{C}+\mathrm{B}) \dot{\psi}^{2} .\right.
\end{aligned}
$$


On the other hand, if the spring factors about $\mathrm{CD}, \mathrm{AB}$ are $R^{-1}, S^{-1}$, the potential energy of the system is given by

$$
2 \mathrm{U}=\mathrm{R}^{-1} \phi^{2}+\mathrm{S}^{-1} \psi^{2} \text {. }
$$

Now let $\quad \theta=90^{\circ}-\beta, \phi=x, \psi=-y$,

and

$$
\mathrm{O}=\mathrm{L}, \mathrm{B}=\mathrm{N}-\mathrm{L}(\mathrm{L}<\mathrm{N}), \mathrm{C} \sin \beta=\mathrm{M} \text {, }
$$

so that

$$
\gamma^{2}=C \sin ^{2} \beta /(B+C) \text {. }
$$

Then the kinetic and potential energies are the same in the two problems, and an exact analogy is established. The charges are represented by the angular displacements of the sphere and the ring.

The defects of this mechanism are the absence of symmetry and the fact that the coupling cannot be carried to 1 unless $\mathrm{L}=\mathrm{N}, \mathrm{B}=0$. Prof. Barton desires a mass $\mathrm{M}$ to represent the mutual induction. This seems to be a mistake. The coupling in the electrical system is introduced not by making any addition but by changing the configuration of the parts. This feature is particularly well illustrated by the effect of altering the angle $\beta$ in the mechanism described.

Dunsink Observatory,

Oct. 27, 1917.

XLIX. Astronomical Consequences of the Electrical Theory of Matter. Supplementary Note by Sir OuIver Lodge* .

T $\mathrm{T}$ is barely possible to expound the position more clearly 1 than Professor Edulington has expounded it in two admirabie papers which have appeared in the September and October issues of the Phil. Mag.; but a summary may be convenient.

It is so unusual to take account of varying mass that traps lie in wait for the unwary, and one is apt to overlook some of the consequences, or to deal with the variations incompletely.

When thoroughly considered, a force which increases speed is opposed by aninertia different from that encountered by a transverse or merely deflecting force, for the longitudinal or tangential force contains the term $v d m / d t$ as well as the term $n d v / d t$.

It is better therefore to deal with momentum throughout, and so avoid the complication of a difference between longitudinal inertia and transverse inertia. (See Note 2.)

* Communicated by the Author. 